\title{
Formal Model for Contract Negotiation in Knowledge-Based Virtual Organizations
}

\author{
Mikołaj Zuzek ${ }^{1}$, Marek Talik ${ }^{1}$, Tomasz Świerczyński ${ }^{1}$, Cezary Wiśniewski ${ }^{1}$, \\ Bartosz Kryza $^{2}$, Łukasz Dutka ${ }^{2}$, and Jacek Kitowski ${ }^{1,2}$ \\ ${ }^{1}$ Institute of Computer Science, AGH-UST, Krakow, Poland \\ 2 Academic Computer Centre CYFRONET AGH, Krakow, Poland \\ \{bkryza, dutka,kito\}@agh.edu.pl
}

\begin{abstract}
In this paper we propose a formal model which supports the process of setting up a Virtual Organization by means of allowing contract negotiation between parties pariticipating in such VO. The negotiated contract is used to configure the necessary Grid middleware components for the purpose of contract enforcement during the lifetime of the Virtual Organization. We present a brief overview of the framework and discuss in detail the formal model underlying the process of contract negotiation and how the ontological description of the domains related to given $\mathrm{VO}$ supports this process.
\end{abstract}

Keywords: Virtual Organization, Contract Negotiation, Ontology, Grid

\section{Introduction}

As Grid systems are being moved from academic and research facilities to more challenging business and commercial applications, such issues as control of resource sharing become of crucial importance. In order to manage and share resources within the Grid the idea of Virtual Organizations emerged, which enables sharing only subsets of resources among partners of such Virtual Organization within a potentially larger Grid setting. In order to support creation and management of such Virtual Organization, Grid middleware must support several aspects such as security, resource sharing policy definition and enforcement, resource discovery and usage limited according to the VO policy and other. We propose a semantic based approach, implemented in the form of a framework, called FiVO (Framework for intelligent Virtual Organization) that supports creation and management of dynamic Virtual Organizations with special focus on authorization of access to resources based on ontologies 12 . In this paper we focus on its contract negotiation component and in particular on the formal model which allows to control and verify the negotiations process and its result. The contract, described by a special ontology, provides all information necessary for configuration of Virtual Organization in a Grid system, by automatically translating proper contract statements to configuration options of such systems as for example VOMS (Virtual Organization Management System) 3] or PERMIS [4. Contracts also allow for specification of non-functional parameters of the envisioned 
VO collaborations, especially SLA's (Service Level Agreements) in order to provide for the Grid monitoring layer necessary data for the contract enforcement [5]. Our work is being evaluated within the EU-IST project Gredia [6], on two commercial applications. First is related to inter-banking solution for automatic credit-scoring of bank users credit requests. The second one is a media application oriented on providing a collaborative environment for nomadic journalists.

\section{Related Work}

The idea of supporting Virtual Organizations with formal model of contract negotiation process is not present in existing works. However, some attempts were already made in order to deal with supporting Virtual Organizations with contract based agreements on how to share the resources of the entities participating in some VO. The authors of [7] describe requirements for automating the contract management in a VO. They identify 3 kinds of contracts in a VO: business contract, ICT contract and ASP (Application Service Provider) contract. In [8] an attempt was made to formalize a definition of contract based multi-agent Virtual Organization. The authors define 4 key properties of VOs: Autonomy, Heterogeneity, Dynamism and Structure. They use terminology from agent-based systems, e.g. they refer to the VO itself as an agent. The contract is defined as a set of commitments, goals and agents in some context. The paper introduces a formal definition of a hierarchical VO with a set of agents (which can be VOs themselves), policies, goals and commitments. The VO is then a set of bilateral contracts between agents in a VO, and can be more easily defined in a distributed setting. For example for 3 partners and 2 contracts $A \leftrightarrow B$ and $B \leftrightarrow C$, A and $\mathrm{C}$ don't event need to know about each other. Another example of contract based VO's is presented in [10]. Authors present web-Pilarcos J2EE based agent framework for managing contract based Virtual Organizations. The contract itself is an object (J2EE EntityBean) and can be in several states such as In-negotiation, Terminated etc. The proposed solution is not based on ontologies, and the metadata reasoning is mentioned briefly. The proposed architecture has many different components - which might make it hard for integration with custom systems - should rather provide a more unified interface based on easily adaptable standards. The paper discusses the basic requirements for a VO contract such as modeling of service behaviour, communication services and some non-functional properties such as QoS. In 9] authors present an event based protocol for decision making in Virtual Organizations, for multi-agent systems. The authors introduce a voting protocol based on RONR (Robert's Rules of Order). Thorough discussion of requirements necessary for a general VO management system can be found in [11.

\section{Contract Negotiation Approach in Gredia}

The GREDIA project aims at the development of a Grid application platform, providing high level support for implementation of Grid business applications concerned with users mobility. This platform is generic in order to combine both 


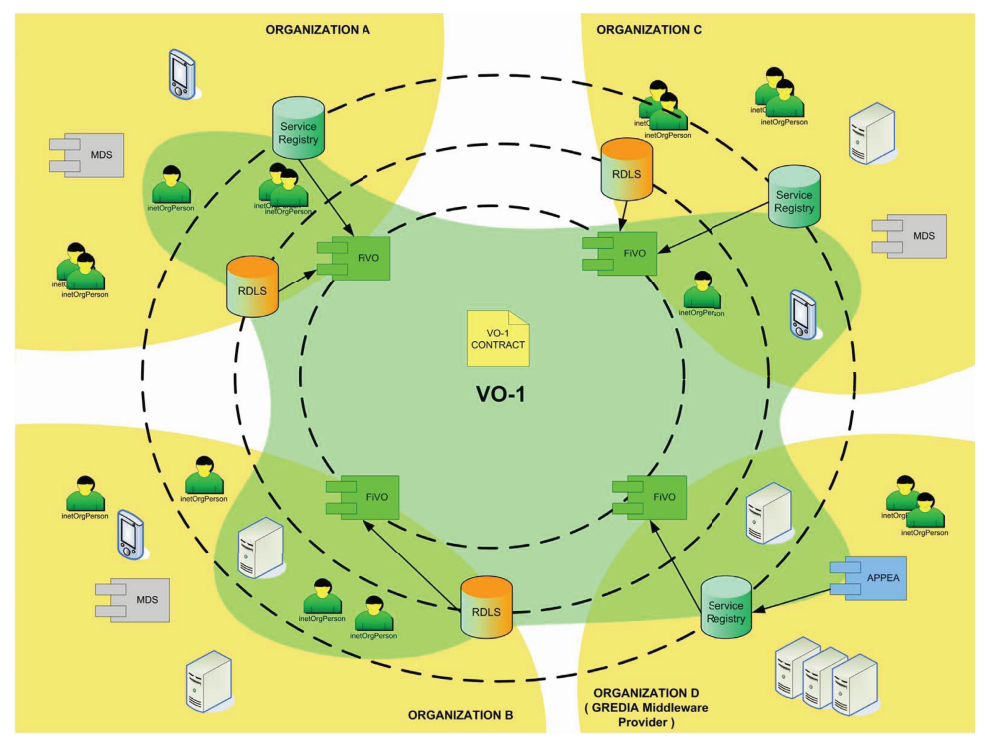

Fig. 1. FiVO overall vision in a distributed Gredia environment

existing and arising Grid middleware, and facilitates the provision of business services, which mainly require access and sharing of large quantities of distributed annotated numerical and multimedia content. One of the main GREDIA features is its focus on mobile users to exploit Grid technologies in a seamless way by enabling mobile access and sharing of distributed content. The potential results of the platform are being validated through two pilot applications, including media and banking. Fig. 1 presents example deployment of the FiVO framework in a distributed Gredia environment. Four organizations are sharing their resources within the VO-1. FiVO component is deployed within each organization and is responsible for storing semantic descriptions of its contents (i.e. resources provided to other organizations). These descriptions can include such aspects of organization as its structure and business logic described in proper ontology as well as hardware, data and service resources available and provide for sharing with other organizations including security and QoS rules. FiVO instances deployed in particular environment can connect through a peer-to-peer overlay, based on Grid Organizational Memory (GOM) knowledge base [12], in order to form a VO and collaborate on emerging tasks. The main feature of FiVO is the support for contract negotiation and management, which enables coordinated establishment of agreement among partners who want to create a new Virtual Organization. In order to enable organizations to define an unambigous agreement on how to share their resources in the form of ontology, the contract ontology provides all the necessary generic concepts, covering such aspects as the notion of Virtual Organization, security and authorization rights, Quality 
of Service in the form of Service Level Agreement statements as well as the negotiation process itself. These generic concepts have to be extended by the domain specific concepts of a particular VO in order to reflect its actual intented goal. The contract itself is simply a set of ontology individuals. Abstract statements from the contract are used to configure the VO middleware, including security and monitoring infrastructure, in order to enforce proper access authorization and QoS, as described by the contract.

\section{Formal Model of Contract Negotiation}

The formal model of contract negotiations in FiVO framework will be presented by the introduction of several definitions. In general we can say, that the contract is a set of statements which state the rules of cooperation between parties, referred to here as agents $(A)$ and the rules specifying how their resources $(X)$ can be shared.

Definition 1. Let $R: A \rightarrow 2^{X}$ be resource ownership function which assigns the sets of resources to agents who own them, with the assumption that agents do not own the same resources at the same time, i.e.:

$$
\forall a_{1}, a_{2} \in A: a_{1} \neq a_{2} \Rightarrow R\left(a_{1}\right) \cap R\left(a_{2}\right)=\emptyset
$$

Definition 2. Atom, $p$, is a logical expression concerning one agent and one of his resources. The set of all possible atoms can be defined as:

$$
\begin{aligned}
& P=\{p=(a, x, z, d): a \in A \wedge x \in R(a) \wedge \\
& \qquad d=\{(u, v): u \in \Omega \wedge v \in \operatorname{Val}(u)\}\}
\end{aligned}
$$

where $a$ is the agent, $x$ is the resource owned by the agent, $z$ represents an action that is supposed to be taken by the agent and $d$ contains the parameters of the action ( $\Omega$ is the set of possible attributes and $V$ al maps the parameters to their possible values).

Definition 3. Statement, $s$, is a set of atoms in the form of a logical sentence. It represents agents' requirement or commitment depending on whether the statement is still under negotiations or is already accepted. All atoms of a given statement must relate to one agent. The set of all possible statements can be defined as:

$$
S=\left\{s=\left(p_{1}, p_{2}, \ldots, p_{n}\right): n \in N_{+} \wedge p_{i}=\left(a, x_{i}, z_{i}, d_{i}\right), i=1,2, \ldots, n\right\}
$$

In order to allow separation of the negotiation process for the whole VO into partial negotiations on subsets of resources that will be included in the overall contract, we introduce the concept of a negotiation table.

Definition 4. The negotiation table is a couple $(O, C)$ of disjunctive sets of statements, where $O$ includes opened statements which are still being negotiatied, 
and $C$ contains already accepted statements (closed). The set of all possible tables can be defined as:

$$
\Phi=\stackrel{\circ}{\Phi} \cup \hat{\Phi}=\left\{(O, C) \in 2^{S} \times 2^{S}: O \cap C=\emptyset\right\}
$$

We say that negotiation table is closed when it does not contain any opened statements, otherwise it is opened. Thus we can define the sets of opened and closed negotiation tables as:

$$
\begin{aligned}
& \stackrel{\circ}{\Phi}=\{(O, C) \in \Phi: O \neq \emptyset\} \\
& \hat{\Phi}=\{(O, C) \in \Phi: O=\emptyset\}
\end{aligned}
$$

Since all negotations take place on at least one negotiation table we can define a negotation state as a subset of the possible negotiations set, i.e.:

Definition 5. The negotiation state is any set of negotiation tables, i.e. $q \subset \Phi$ The changes in the negotiation tables can only be achieved by means of sending proper messages. These messages define who states what about which resources:

Definition 6. By message, $m$, we mean a quintuple defined as:

$$
(a, I, Y, U, R) \in M=A \times M_{I} \times M_{Y} \times M_{U} \times 2^{\stackrel{\circ}{\Phi}}
$$

where a represents the agent who sends the message, I is the set of pairs tablestatement, which specifies which statements should be added to which negotiation table $\left(M_{I}\right)$, thus:

$$
M_{I}=\{I \subset \stackrel{\circ}{\Phi} \times S:((O, C), s) \in I \Rightarrow s \notin O \cap C\}
$$

The above definition disallows adding statements which are already in a given negotiation table. $Y$ is the set of statements accepted by the agent (only statements already added to the given negotiation table can be accepted), i.e:

$$
M_{Y}=\{Y \subset \stackrel{\circ}{\Phi} \times S:((O, C), s) \in Y \Rightarrow s \in O\}
$$

$U$ specifies the set of statements updated by the current message and it contains triples, which contain the set on which the statement is updated, statement already existing in this table and the new statement which does not yet exist in this table:

$$
M_{U}=\left\{U \subset \stackrel{\circ}{\Phi} \times S \times S:\left((O, C), s_{1}, s_{2}\right) \in U \Rightarrow s_{1} \in O \wedge s_{2} \notin O \cup C\right\}
$$

The statement from the set $U$ can only modify parameters (d) of existing statements, thus the following holds:

$$
\left((O, C),\left(a_{1}, x_{1}, z_{1}, d_{1}\right),\left(a_{2}, x_{2}, z_{2}, d_{2}\right)\right) \in U \Rightarrow a_{1}=a_{2} \wedge x_{1}=x_{2} \wedge z_{1}=z_{2}
$$


Finally the set $R$ is the set of tables rejected by the agent. It cannot contain already closed negotiation tables, as well as it cannot contain the sets mentioned by statements in $I, Y$ and $U$, thus we have:

$$
((O, C), s) \in I \vee((O, C), s) \in Y \vee\left((O, C), s_{1}, s_{2}\right) \in U \Rightarrow(O, C) \notin R
$$

Since agent cannot accept statements which are not related to him, we have:

$$
(a, I, Y, U, R) \in M \wedge((O, C),(\tilde{a}, \tilde{x}, \tilde{z}, \tilde{d})) \in Y \Rightarrow a=\tilde{a}
$$

Now, we can use these definitions to verify that a message is valid with respect to the negotiation state:

Definition 7. Let $V: 2^{\Phi} \rightarrow M$ be a mapping, such that:

$$
\begin{aligned}
m= & (a, I, Y, U, R) \in V(q) \Leftrightarrow \\
& {\left[((O, C), s) \in Y \vee\left((O, C), s_{1}, s_{2}\right) \in U \vee(O, C) \in C\right] \Rightarrow(O, C) \in q }
\end{aligned}
$$

In a given negotiations state, as correct messages from the set $V(q)$, we mean those, which accept or modify statements from the negotiation tables which are actually contained within that negotiation state. Invalid messages, are those, which relate to statements not present in any of the negotiation tables for the given state.

Definition 8. Let $Q:\left\{(q, m) \in 2^{\Phi} \times M: m \in V(q)\right\} \rightarrow 2^{\Phi}$ be a transfer function which assigns to every negotiation state and message the consecutive state. Assuming $m=(a, I, Y, U, R)$ we get:

$$
\begin{aligned}
Q(q, m)= & q \cup(\underset{((O, C), s) \in I}{\cup}(O \cup\{s\}, C)) \backslash(\underset{((O, C), s) \in Y}{\cup}(O, C)) \cup \\
& (\underset{((O, C), s) \in I}{\cup}(O \backslash\{s\}, C \cup\{s\})) \backslash(\underset{((O, C), s) \in Y}{\cup}(O, C)) \cup \\
\left(\underset{\left((O, C), s_{1}, s_{2}\right) \in U}{\cup}\right. & \left.\left.\left.\cup \cup\left\{s_{2}\right\} \backslash\left\{s_{1}\right\}, C\right)\right) \backslash \underset{\left((O, C), s_{1}, s_{2}\right) \in U}{\cup}(O, C)\right) \backslash C
\end{aligned}
$$

The message modifies the negotiation state according to these rules:

- statements of the form $((O, C), s)$ from set $I$ create new statements $s$ in the negotiation table $(O, C)$, so the negotiation table changes to $(O \cup\{s\}, C)$

- statements of the form $((O, C), s)$ from set $Y$ change the statement to close, i.e. it moves them from the set $O$ to set $C:(O \backslash\{s\}, C \cup\{s\}$

- statements of the form $\left((O, C), s_{1}, s_{2}\right)$ from the set $U$, cause replacement of the statement $s_{1}$ with statement $s_{2}$ within the table $(O, C)$, thus: $\left(O \cup\left\{s_{2}\right\} \backslash\right.$ $\left.\left\{s_{1}\right\}, C\right)$

- negotiation tables from the set $C$ are removed from the input state

Each valid message sent to a negotiation table, modifies the state of the negotiation process. Thus we can define a mapping which gives us the negotiation state from the sequence of messages: 
Definition 9. Let us define a mapping $\varphi: D_{\varphi} \rightarrow 2^{\Phi}$, such that:

$$
\begin{gathered}
\emptyset \in D_{\varphi} \\
\varphi(\emptyset)=\emptyset \\
\underset{i=0,1,2, \ldots, n+1}{\forall} m_{i} \in M \wedge \Psi=\left(m_{0}, \ldots, m_{n}\right) \wedge \tilde{\Psi}=\left(m_{0}, \ldots, m_{n+1}\right) \wedge \Psi \in D_{\varphi} \\
\Rightarrow\left[m_{n+1} \in V(\varphi(\Psi)) \Rightarrow \tilde{\Psi} \in D_{\varphi} \wedge \varphi(\tilde{\Psi})=Q\left(\varphi(\Psi), m_{n+1}\right)\right]
\end{gathered}
$$

The above mapping maps sequences of messages into negotiation states. An empty sequence is assigned an empty negotiation state. If a given negotiation sequence $\Psi$ belongs to the domain of state function (which means it represents correct negotiation process) then the value of function $\varphi(\Psi)$ is the state of negotiations after applying all messages from this function.

Definition 10. Negotiations is any finite sequence of messages which belongs to the domain of state function $\varphi$.

We say that negotiations completed successfully when the last state contains at least one closed negotiation table and does not contain any opened negotiation tables. Thus finally we can define a contract:

Definition 11. A contract is a set of statements contained in the final negotiations state. i.e.:

$$
K=\underset{(O, C) \in \varphi(\Psi)}{\cup} C
$$

The definitions presented above specify formal way of negotiating a contract for a $\mathrm{VO}$, therefore $\mathrm{VO}$ can be understood as a practical realization of the above defined contract. This allows to verify that the negotiations were fair and that they completed successfully, i.e. all parties were finally satisfied. Another step is to use the definition of a VO goal, defined as a set of requirements for the contract to automatically verify, whether all initial requirements for the VO were satisfied. In order to support dynamic VOs, where the conditions can change during the operation of $\mathrm{VO}$, the contract, as defined above can be ammended, by starting the negotiations again with the current state of the contract as the initial state.

\section{From Contract Model to VO Ontology}

The formal model provides means to implement a negotiation framework which can allow parties to define the rules of cooperation within a given Virtual Organization. After the contract is successfuly negotiated, the framework should ensure the rules agreed upon are obeyed. According to the presented formal model a sample statement from the EasyLoan banking Virtual Organization looks like below: 
$s=$ StCreditCalculation $=$

$\{p=(a=$ '\#Marco', $x=$ '\#CreditCalculationService', $z=$ '\#ProvidesService', $d=\{($ \#TimeToComplete', '15minutes'), ('\#accessRole','\#BankClerk')\})\}

These statements are encoded in the Web Ontology Language with respect to proper contract ontology, and the above statement in this ontology is rendered as:

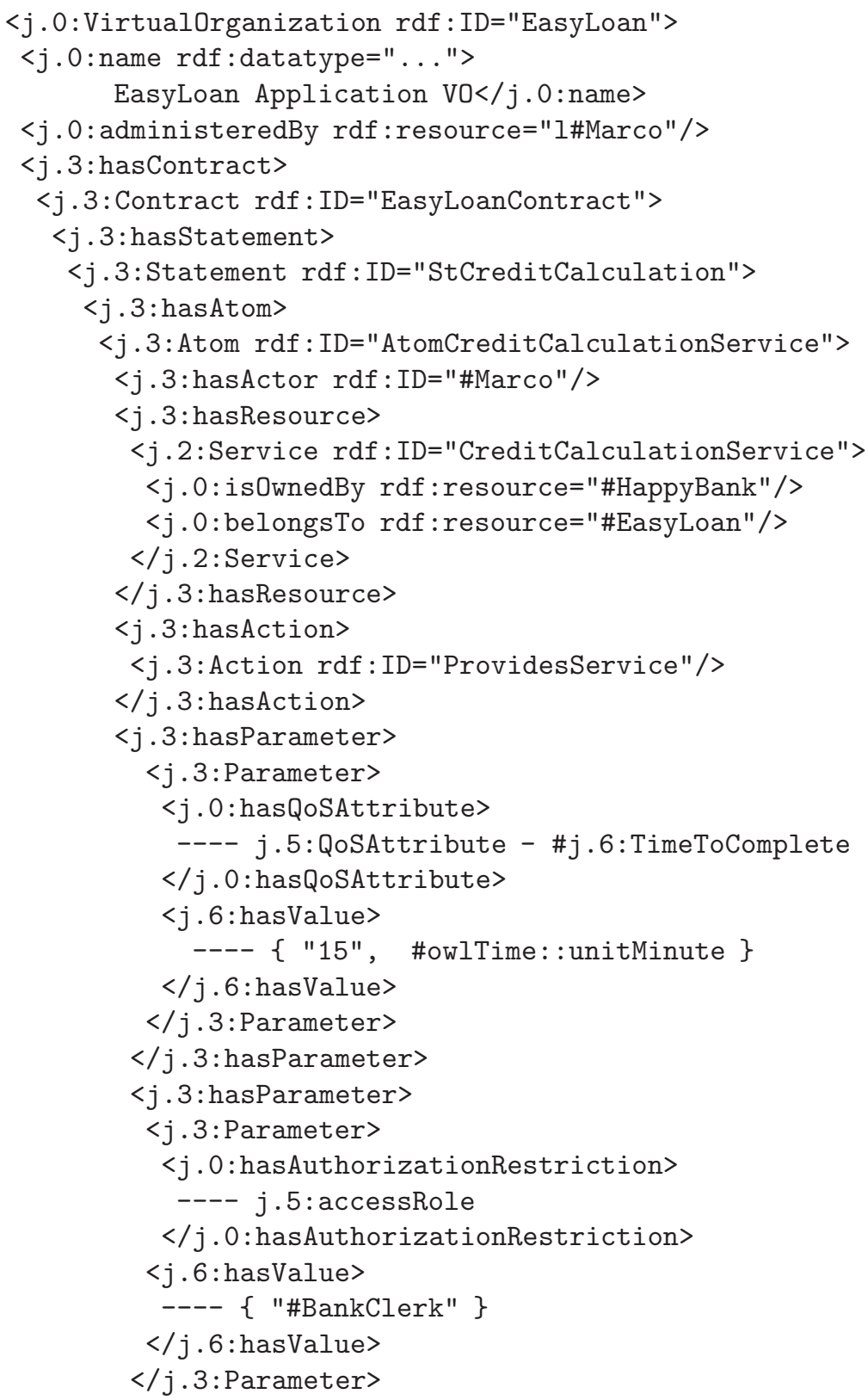




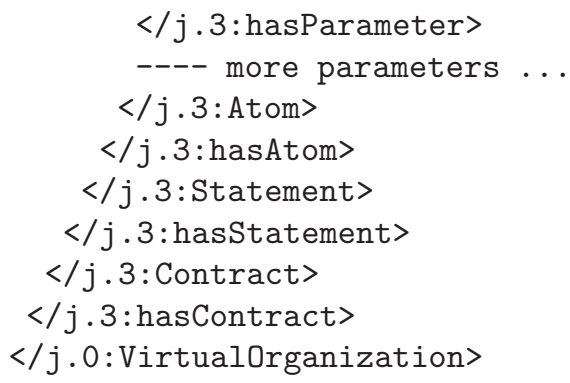

In Fig. 2, we can see sample contract ontology visualization for EasyLoan Virtual Organization of the banking scenario.

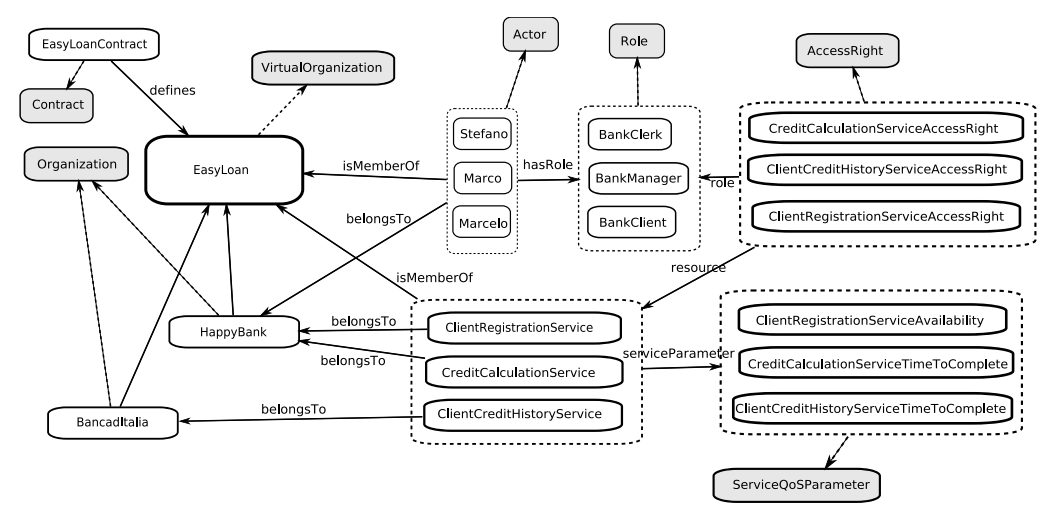

Fig. 2. Graphical representation of ontological contract for Banking application

This contract is used by FiVO to configure Grid middleware services such as VOMS, PERMIS or MDS in order to actually deploy the Virtual Organization in the Grid environment. Further enforcement of the contract statements is performed automatically by these services.

\section{Conclusions and Future Work}

In this paper we have presented the vision and architecture of the FiVO (Framework for Intelligent Virtual Organizations) which enables contract negotiation and management for Grid-based Virtual Organizations in a business setting. We believe that such functionality will foster the adoption of Grid and Virtual Organizations in commercial applications by simiplifying the process of Virtual Organization inception and management of agreements on how the resources of each participating organizations should be shared among partners of a VO. The framework is currently being implemented and the future work will include, development of ontologies required for contract definition and evaluation of the framework on pilot applications. The formal model will be extended with a 
equivalence relation by means of the type of resources and allowing OR operator between atoms in a statement.

Acknowledgements. The authors want to acknowledge the support of the EU Gredia Project (IST-FP6-034363) and AGH University of Science and Technology grants 11.11.120.777 and 500-08.

\section{References}

1. Kryza, B., Dutka, L., Slota, R., Kitowski, J.: Supporting knowledge-based dynamic virtual organizations with contracts. In: Proc. of eChallenges 2007 Conference and Exhibition, The Hague, Netherlands, October 24-26, 2007, pp. 937-945 (2007)

2. Kryza, B., Dutka, L., Slota, R., Pieczykolan, J., Kitowski, J.: Gvosf: Grid virtual organization semantic framework. In: Bubak, M., Turala, M., Wiatr, K. (eds.) Proc. of Cracow Grid Workshop 2006 (CGW 2006), pp. 104-110. ACK-Cyfronet AGH, Krakow (2007)

3. Alfieri, R., Cecchini, R., Ciaschini, V.: Voms, an authorization system for virtual organizations. In: Fernández Rivera, F., Bubak, M., Gómez Tato, A., Doallo, R. (eds.) Across Grids 2003. LNCS, vol. 2970, pp. 33-40. Springer, Heidelberg (2004)

4. Chadwick, D.W., Otenko, A.: The permis x.509 role based privilege management infrastructure. Future Generation Comp. Syst. 19, 277-289 (2003)

5. Alipio, P., Neves, J., Carvalho, P.: An ontology for network services. Computing and Informatics 26(5), 543-561 (2007)

6. Gredia Consortium: Gredia project website, http://www.gredia.eu

7. Shelbourn, M., Hassan, T., Carter, C.: 3.1. Legal and contractual framework for the VO. In: Camarinha-Matos, L.M., Afsarmanesh, H., Ollus, M. (eds.) Virtual Organizations Systems and practices, pp. 167-176. Springer, Heidelberg (2005)

8. Udupi, Y.B., Singh, M.P.: Contract enactment in virtual organizations: A commitment-based approach. In: Proc. of the Twenty-First National Conference on Artificial Intelligence and the Eighteenth Innovative Applications of Artificial Intelligence Conference, Boston, Massachusetts, USA, July 16-20, 2006. AAAI Press, Menlo Park (2006)

9. Pitt, J.V., Kamara, L., Sergot, M.J., Artikis, A.: Formalization of a voting protocol for virtual organizations. In: 4rd International Joint Conference on Autonomous Agents and Multiagent Systems (AAMAS 2005), Utrecht, The Netherlands, July 25-29, 2005, pp. 373-380. ACM, New York (2005)

10. Metso, J., Kutvonen, L.: Managing virtual organizations with contracts. In: Workshop on Contract Architectures and Languages (CoALa 2005) (2005)

11. Ratti, R., Camarinha-Matos, L.M., et al.: Specification of vo creation support tools. Technical report, Ecolead Consortium (2006)

12. Kryza, B., Slota, R., Majewska, M., Pieczykolan, J., Kitowski, J.: Grid Organizational Memory - Provision of a High-level Grid Abstraction Layer Supported by Ontology Alignment. Future Generation Computer Systems (FGCS) 23(3) (March 2007) 